\title{
Design and Simulation of a Circularly Polarized Square Horn Antenna
}

\author{
Song Lizhong ${ }^{1, \text { a }}$, Cao Hongwei ${ }^{1}$ and Yin Weiwei ${ }^{1}$ \\ ${ }^{1}$ School of Information and Electrical Engineering, Harbin Institute of \\ Technology at Weihai, Weihai, 264209, P. R. China \\ aemail:songlizhong@hitwh.edu.cn
}

\begin{abstract}
This paper designed a circularly polarized (CP) horn antenna working at X frequency band in this paper. The stepped septum polarizer (SP) was used to convert the linear polarization into circular polarization for the discussed square horn antenna. The circularly polarized horn antenna designed in this paper was simulated by using the full wave electromagnetic simulation software, and the specific structure and parameters were provided. The voltage standing wave ratio (VSWR) was lower than 2 within the frequency range of from $8 \mathrm{GHz}$ to $8.25 \mathrm{GHz}$. The simulated axial ratio (AR) at the boresight direction at frequency $8 \mathrm{GHz}$ was about 3dB. The simulated gains of the designed antenna were about higher than $17 \mathrm{dBi}$ within the frequency range of $8 \mathrm{GHz}$ to $8.2 \mathrm{GHz}$. The design and simulation results of circularly polarized horn antenna in this paper can provide a reference for the practical engineering application.
\end{abstract}

Keywords: horn antenna, circular polarization, septum polarizer, antenna simulation.

\section{Introduction}

Circularly polarized (CP) antennas have been widely applied in modern satellite communications and radar systems because of many advantages[1-2]. In satellite and ground station, $\mathrm{CP}$ avoids the Faraday rotation effect. According to the antenna theory, the handedness of $\mathrm{CP}$ wave is reversed when it is reflected off a mirror, so the reflected wave can not be received by the transmitting antenna. The CP antenna has the advantages of restraining interference from rain and fog, suppressing multipath effect. There are a lot of kinds of circularly polarized antennas such as horn antenna, microstrip antenna, helix antenna and waveguide slot antenna. The circularly polarized horn antennas are usually used in communication systems and radar systems because of simple structures and their good performances of high power-handling capability, ease of excitation, high gain, and moderate bandwidth[3]. Generally the horn antenna can be turned into a circularly polarized antenna by using a suitable polarizer. In reference [4], a $\mathrm{X}$-band double circularly polarized horn antenna based on waveguide circular polarizer was introduced and the coaxial multimode horn of two chokes was used 
to suppress the sidelobe. In reference [5], the septum polarizer was studied by using the mode matching and finite element hybrid method. In reference [6], a kind of circularly polarized waveguide phased array antenna loaded with the dielectric material of K-band was designed and the circular signal was produced by step wall shifter which was arranged inside the waveguide antenna. In this paper, a circularly polarized square horn with stepped septum was designed and simulated. The center frequency was $8 \mathrm{GHz}$. The simulated performances of the designed horn antenna were provided.

\section{Antenna structure and Design}

The horn antenna designed in this paper was composed of coaxial-waveguide converter, waveguide circular polarizer and horn. The structure of the discussed circularly polarized horn antenna can be shown in Fig.1.

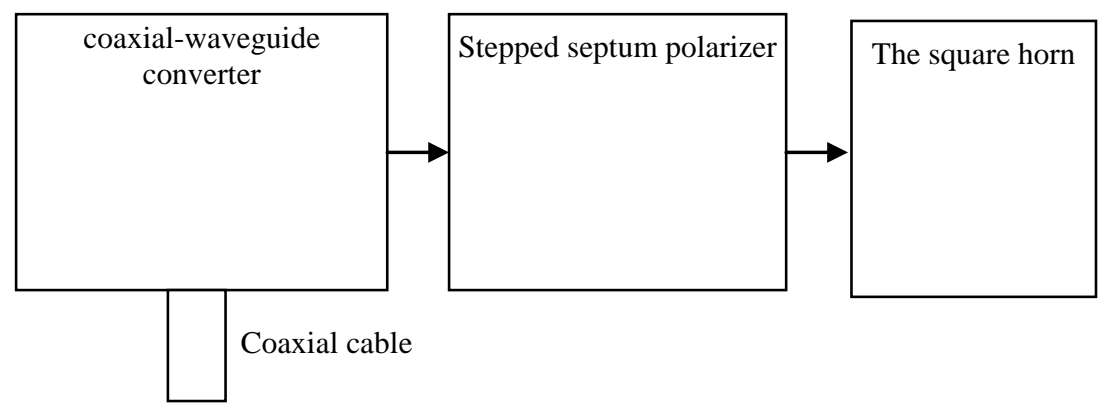

Fig.1: The structure of the discussed circularly polarized horn antenna

The model of designed horn antenna is shown in Fig. 2 and the total dimension of the horn antenna is shown in Fig.3. In this paper, the side length of square horn aperture was 190 millimeters and total length at axial direction of the horn was 281 millimeters, and the values of these two parameters were determined according to the requirements of practical engineering. The distance between the feeding point and the end of squre waveguide was 13 millimeters, and this parameter was obtained by using the simulation and optimization through the full wave electromagnetic simulation software. The diameter of feeding probe was 2.54 millimeters. The side length of square waveguide was 35.9 millimeters, which was deternined by the working frequency. 

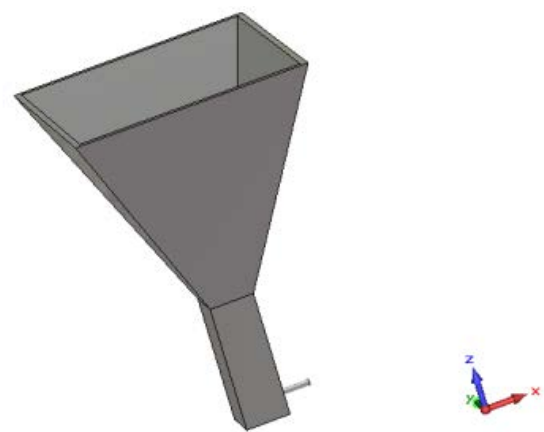

(a)The total mode view

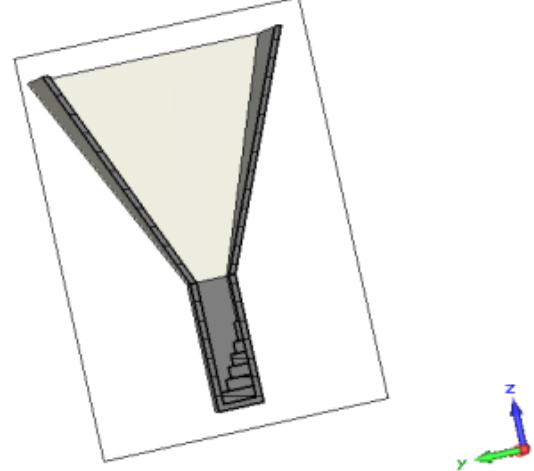

(b) The model view using cutting plane Fig. 2 The model of designed horn antenna
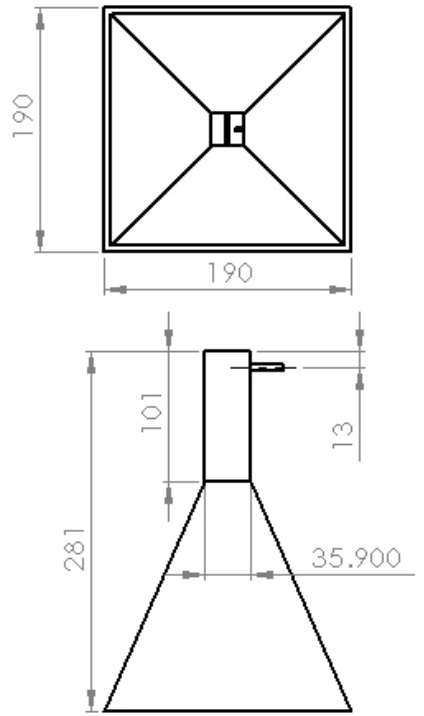

Fig.3 the total dimension of the horn antenna 
The septum circular polarizer is an important circular polarizer which is usually used in the waveguide network. The advantages of septum circular polarizer include simple structure, good isolation and low VSWR. What's more, it is easy to connect the septum circular polarizer with waveguide antenna. In this paper, a stepped septum polarizer was employed, which was inserted into a square waveguide. The stepped septum polarizer was directively connected with a square horn. The dimensions of designed stepped septum polarized were shown in Fig.4.

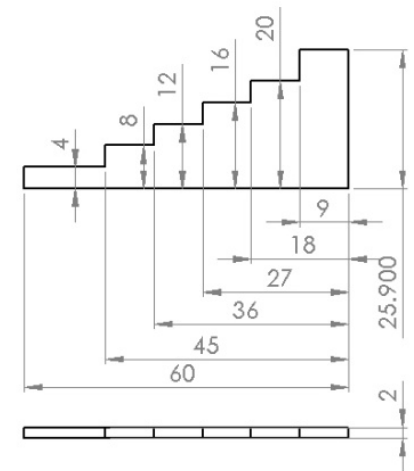

Fig.4 The structure and dimensions of the stepped septum poalrizer

\section{Simulation results}

The designed circularly polarized square horn antenna was simulated and optimized by using CST. The specific simulation results were provided in this section.Fig.5 showed the simulated VSWR of the designed horn antenna. The simulated VSWR was lower than 2 within the frequency range between $8 \mathrm{GHz}$ and $8.25 \mathrm{GHz}$. The approximately minimum VSWR appeared at the frequency of $8.15 \mathrm{GHz}$

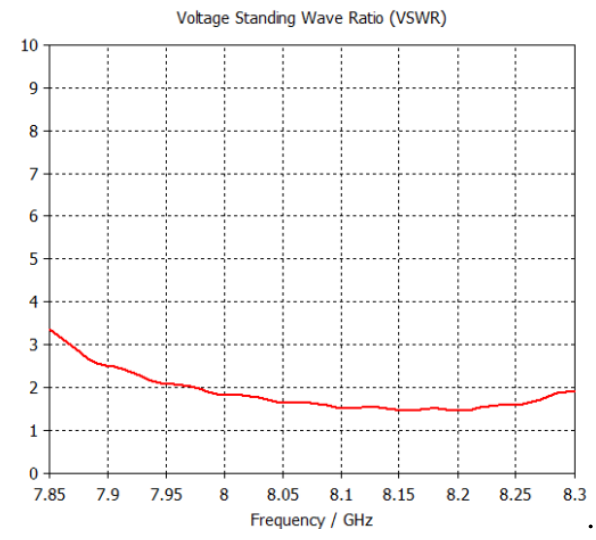

Fig.5: The simulated VSWR of the designed horn antenna 
In this section, the radiation patterns of the designed horn antenna were provided and discussed. The discussed frequency range was from $8 \mathrm{GHz}$ to 8.2GHz. The simulated results of radiation patterns at $8 \mathrm{GHz}$ were shown in Fig.6. Fig.6 (a) and Fig.6 (b) were the Gain patterns at $x O z$ plane and yoz plane, respectively. The Gain of the designed horn antenna was about $18.1 \mathrm{dBi}$, and the half power beam widths at $\mathrm{xOz}$ plane and yoz plane were 22.5 degree and 20.5 degree, respectively. The observed $\mathrm{AR}$ at the boresight direction for the frequency of $8 \mathrm{GHz}$ was lower than $3 \mathrm{~dB}$, so the circularly polarized radiation performance was realized.

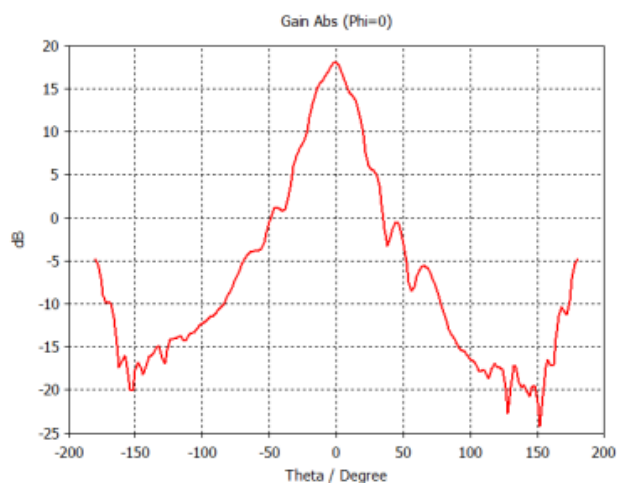

(a)Pattern at $x O z$ plane

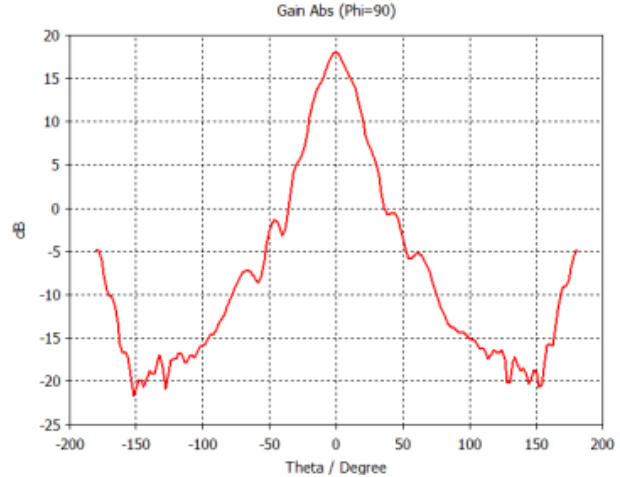

(b) Pattern at yoz plane

Fig. 6 The simulated results of radiation patterns at $8 \mathrm{GHz}$

The simulated results of radiation patterns at $8.1 \mathrm{GHz}$ were shown in Fig.7. The Gain of the designed horn antenna at $8.1 \mathrm{GHz}$ was about $18 \mathrm{dBi}$, and the half power beam widths at xoz plane and yoz plane were 23.5 degree and 20.6 degree, respectively. The observed AR at the boresight direction for the frequency of $8.1 \mathrm{GHz}$ was about $4 \mathrm{~dB}$, which indicated the circularly polarized radiation performance slightly degraded for the frequency of $8.1 \mathrm{GHz}$. 


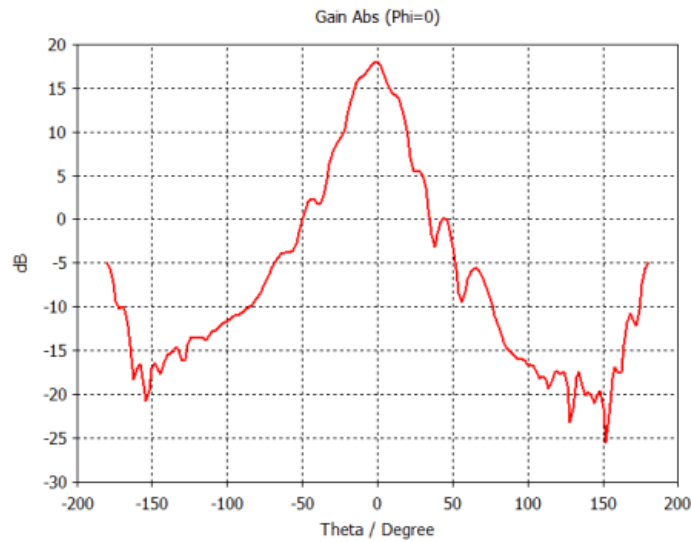

(a)Pattern at $x o z$ plane

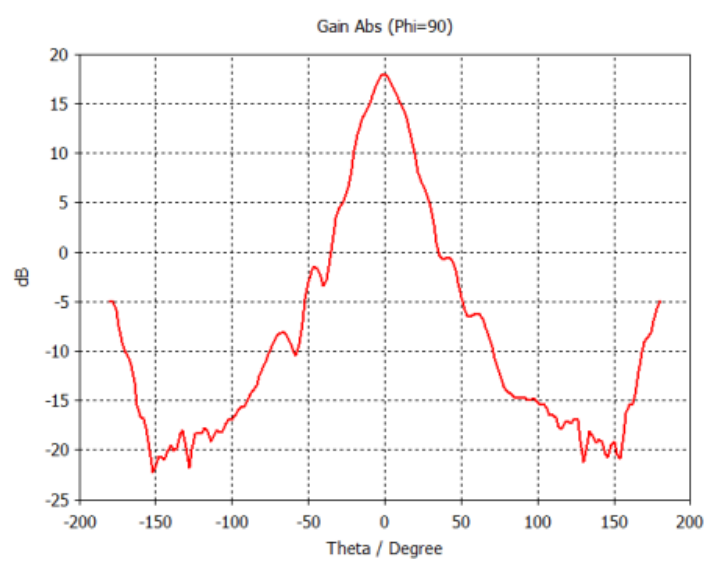

(b) Pattern at yoz plane

Fig.7 The simulated results of radiation patterns at $8.1 \mathrm{GHz}$

The simulated results of radiation patterns at $8.2 \mathrm{GHz}$ were shown in Fig.8. The Gain of the designed horn antenna at $8.1 \mathrm{GHz}$ was about $17.9 \mathrm{dBi}$, and the half power beam widths at xoz plane and yoz plane were 23.1 degree and 21 degree, respectively. According to simulation results, the observed gains and radiation patterns were steady for the frequency range from $8 \mathrm{GHz}$ to $8.2 \mathrm{GHz}$. The observed $\mathrm{AR}$ at the boresight direction for the frequency of $8.2 \mathrm{GHz}$ was lower than $5 \mathrm{~dB}$, and it can be seen that the AR increased when the frequency increased for the designed circularly polarized horn antenna. 


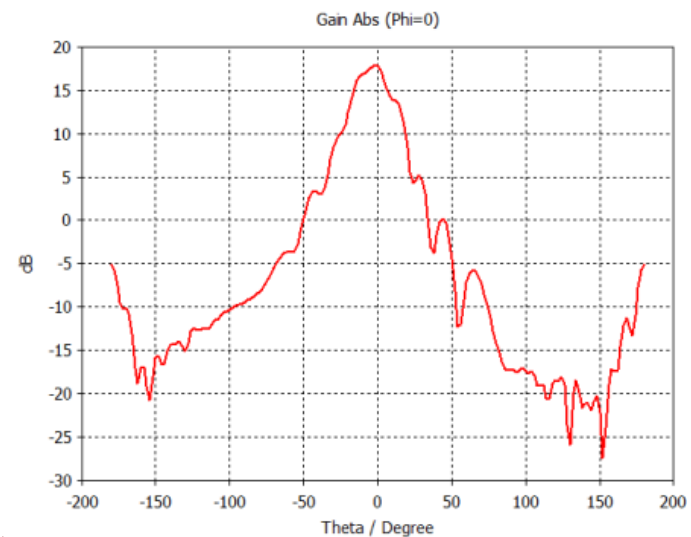

( a)Pattern at $x o z$ plane

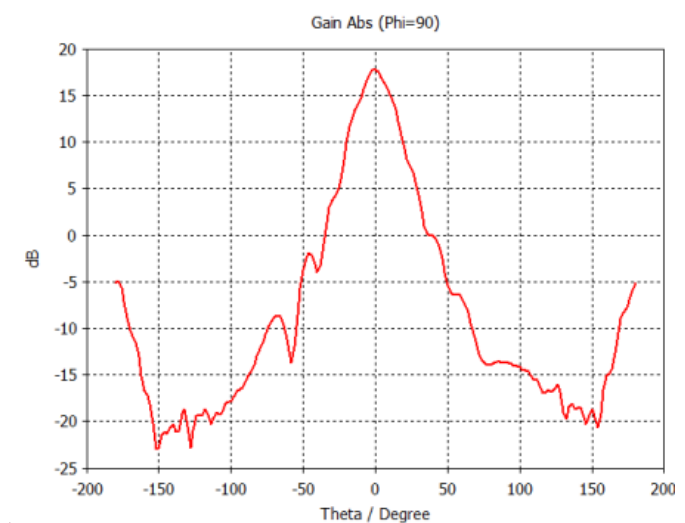

(b) Pattern at yoz plane

Fig. 8 The simulated results of radiation patterns at $8.2 \mathrm{GHz}$

\section{Conclusions}

A circularly polarized square horn antenna was designed and simulated by using the electromagnetic simulation software in this paper. The designed horn antenna was constructed based on the traditional stepped septum polarizer. The square horn aperture was employed to radiate the circularly polarized electromagnetic waves. The specific design parameters of discussed horn antenna were determined by simulation and optimization. The radiation performances of the designed horn antenna within the operational frequency range were given, which can meet the requirement at several practical applications.

\section{Acknowledgements}

The research work was supported by the National Natural Science Foundation of China (61171181), Project funded by China Postdoctoral Science Foundation 
(2014M561554) and the Astronautical Supporting Technology Foundation of China (2013-HT-HGD06).

\section{References}

[1] Y.J. Sung , Y.-S. Kim. Circular polarised microstrip patch antennas for broadband and dual-band operation. Electronics Letters, Vol. 40, No. 9, pp.520-522, April 2004.

[2] Christopher T. Rodenbeck, Ming-Yi Li, and Kai Chang. Circular-Polarized Reconfigurable Grating Antenna for Low-Cost Millimeter-Wave Beam-Steering. IEEE Transactions on Antennas and Propagation, vol. 52, no. 10, pp. 2759-2764, October 2004.

[3] Chin Yeng Tan, Krishnasamy T. Selvan. A Circularly Polarized Conical Horn with an Inserted Metallic Cone-Sphere. IEEE Antennas and Propagation Magazine, Vol. 55, No.3, pp. 146 - 154,June 2013

[4] Qi Jian. Design and Simulation of X-band double Circularly Polarized Horn Antenna. Aero Weaponry, no.1, pp.38-40,47, February, 2012.

[5] WANG Jia-qi, ZHEN Shu-chun. The Analysis of the Septum Polarizer by Using the MM /FE Method. Modern Radar, vol.23, no.4, pp. 79-81, August 2001.

[6]Han Guodong, Kang Yiding, Wu Wei, DuBiao and Chen Rushan. A study on K-band circularly polarized phased array antenna. Modern Radar, vol.35, no.12, pp.60-63, December 2013. 